\title{
The Borwein conjecture and partitions with prescribed hook differences
}

\author{
David M. Bressoud
}

Submitted: March 10, 1995; Accepted: May 11, 1995

Dedicated to Dominique Foata: teacher, mentor, and friend

\begin{abstract}
Peter Borwein has conjectured that certain polynomials have non-negative coefficients. In this paper we look at some generalizations of this conjecture and observe how they relate to the study of generating functions for partitions with prescribed hook differences. A combinatorial proof of the generating function for partitions with prescribed hook differences is given.
\end{abstract}

\section{Introduction}

In a personal communication to George Andrews in 1990, Peter Borwein made the following three conjectures. We use the notation

$$
\begin{aligned}
(a ; q)_{n} & =\prod_{j=0}^{n-1}\left(1-a q^{j}\right), \\
{\left[\begin{array}{l}
N \\
M
\end{array}\right] } & =\frac{\left(q^{N-M+1} ; q\right)_{M}}{(q ; q)_{M}} .
\end{aligned}
$$

Conjecture 1 The polynomials $A_{n}(q), B_{n}(q)$, and $C_{n}(q)$ defined by

$$
\left(q ; q^{3}\right)_{n}\left(q^{2} ; q^{3}\right)_{n}=A_{n}\left(q^{3}\right)-q B_{n}\left(q^{3}\right)-q^{2} C_{n}\left(q^{3}\right)
$$

have non-negative coefficients.

Conjecture 2 The polynomials $A_{n}^{*}(q), B_{n}^{*}(q)$, and $C_{n}^{*}(q)$ defined by

$$
\left(q ; q^{3}\right)_{n}^{2}\left(q^{2} ; q^{3}\right)_{n}^{2}=A_{n}^{*}\left(q^{3}\right)-q B_{n}^{*}\left(q^{3}\right)-q^{2} C_{n}^{*}\left(q^{3}\right)
$$

have non-negative coefficients. 
THE ELECTRONiC JOURNAL OF COMBINATORICS 3 (2) (1996), \#R4

Conjecture 3 The polynomials $A_{n}^{\star}(q), B_{n}^{\star}(q), C_{n}^{\star}(q), D_{n}^{\star}(q)$ and $E_{n}^{\star}(q)$ defined by

$$
\begin{aligned}
& \left(q ; q^{5}\right)_{n}\left(q^{2} ; q^{5}\right)_{n}\left(q^{3} ; q^{5}\right)_{n}\left(q^{4} ; q^{5}\right)_{n} \\
& \quad=A_{n}^{\star}\left(q^{5}\right)-q B_{n}^{\star}\left(q^{5}\right)-q^{2} C_{n}^{\star}\left(q^{5}\right)-q^{3} D_{n}^{\star}\left(q^{5}\right)-q^{4} E_{n}^{\star}\left(q^{5}\right)
\end{aligned}
$$

have non-negative coefficients.

George Andrews [1] has generalized the first two conjectures:

Conjecture 4 For $m \geq 1$, the polynomials $A^{\dagger}(m, n, t, q), B^{\dagger}(m, n, t, q)$, and $C^{\dagger}(m, n, t, q)$ defined by

$$
\begin{aligned}
\left(q ; q^{3}\right)_{m}\left(q^{2} ; q^{3}\right)_{m}\left(z q ; q^{3}\right)_{n}\left(z q^{2} ; q^{3}\right)_{n} & \\
= & \sum_{t=0}^{2 n} z^{t}\left[A^{\dagger}\left(m, n, t, q^{3}\right)-q B^{\dagger}\left(m, n, t, q^{3}\right)-q^{2} C^{\dagger}\left(m, n, t, q^{3}\right)\right]
\end{aligned}
$$

have non-negative coefficients.

Dennis Stanton has discovered a generalization of the first conjecture. We can use the $q$-binomial theorem to expand ( $k$ odd, $1 \leq a<k / 2$ )

$$
\left(q^{a} ; q^{k}\right)_{m}\left(q^{k-a} ; q^{k}\right)_{n}=\sum_{\nu=(1-k) / 2}^{(k-1) / 2}(-1)^{\nu} q^{k\left(\nu^{2}+\nu\right) / 2-a \nu} F_{\nu}\left(q^{k}\right)
$$

where

$$
F_{\nu}(q)=\sum_{j=-\infty}^{\infty}(-1)^{j} q^{j\left(k^{2} j+2 k \nu+k-2 a\right) / 2}\left[\begin{array}{c}
m+n \\
m+\nu+k j
\end{array}\right] .
$$

Each monomial in $q^{k\left(\nu^{2}+\nu\right) / 2-a \nu} F_{\nu}\left(q^{k}\right)$ involves a power of $q$ for which the exponent is congruent to $-a \nu$ modulo $k$.

Conjecture 5 If a is relatively prime to $k$ and $m=n$, then the coefficients of $F_{\nu}(q)$ are non-negative.

The polynomial $F_{\nu}(q)$ appears to be a special case of the generating function for partitions "with prescribed hook differences," [2]. In particular, it is shown in that paper that if $\alpha+\beta<2 K$ and $-K+\beta \leq n-m \leq K-\alpha$, then

$$
G(\alpha, \beta, K ; q)=\sum_{j}(-1)^{j} q^{j[K(\alpha+\beta) j+K(\alpha-\beta)] / 2}\left[\begin{array}{c}
m+n \\
m+K j
\end{array}\right]
$$

is the generating function for partitions inside an $m \times n$ rectangle with "hook difference conditions" specificed by $\alpha, \beta$, and $K$. The polynomial $F_{\nu}(q)$ is simply the special case

$$
K=k, \quad \alpha=\nu+\frac{k+1}{2}-\frac{a}{k}, \quad \beta=-\nu+\frac{k-1}{2}+\frac{a}{k} .
$$


Since we know that this is a generating function, it follows that the coefficients are non-negative.

The only problem with this analysis is that the hook difference conditions defined in [2] only make sense for integer values of $\alpha, \beta$, and $K$. In section 2 , we will examine these hook difference conditions, and in section 3 we will consider what is involved in extending the definition to non-integer values. We are not able to show that $F_{\nu}(q)$ is a generating function. However, it is possible to construct a family of partition generating functions, $\mathcal{A}_{m, n}(q)$, that are remarkably close to $A_{n}(q)$ when $m=n$. Furthermore, it appears that conjecture 5 can be strengthened to the following.

Conjecture 6 Let $\alpha$ and $\beta$ be positive rational numbers and $K$ an integer greater than 1 such that $\alpha K$ and $\beta K$ are integers. If $1 \leq \alpha+\beta \leq 2 K-1$ (with strict inequalities when $K=2$ ) and $-K+\beta \leq n-m \leq K-\alpha$, then $G(\alpha, \beta, K ; q)$ has non-negative coefficients.

This conjecture is justified heuristically by the arguments of section 3. Several special cases have been verified. For $K=2$ and $m=n$, this author has proven identities that imply the conjecture for $\alpha=1, \beta=1 / 2$ and $\alpha=3 / 2$, $\beta=1[4]:$

$$
\begin{aligned}
& G(1,1 / 2,2 ; q)=\sum_{j=0}^{m} q^{j m}\left[\begin{array}{c}
m \\
j
\end{array}\right], \\
& G(3 / 2,1,2 ; q)=\sum_{j=0}^{m} q^{j^{2}}\left[\begin{array}{c}
m \\
j
\end{array}\right] .
\end{aligned}
$$

Mourad Ismail and Dennis Stanton [5] have proven that the conjecture holds if

$$
\alpha+\beta=K \quad \text { and } \quad \alpha-\beta=m-n+1 .
$$

Experimentally, it appears that the bounds on $n-m$ are sharp. For example, $A_{n}(q)=G(5 / 3,4 / 3,3 ; q)$ with $m=n$. The conjecture states that $G(5 / 3,4 / 3,3 ; q)$ has non-negative coefficients when $|n-m| \leq 1$ : 


$$
\begin{aligned}
& m \quad n \quad G(5 / 3,4 / 3,3 ; q) \\
& 2 \quad 1 \quad 1+q+q^{2} \\
& 21+q+2 q^{2}+q^{3}+q^{4} \\
& 31+q+2 q^{2}+2 q^{3}+2 q^{4}+q^{6} \\
& 41+q+2 q^{2}+2 q^{3}+3 q^{4}+q^{5}+q^{6}-q^{9}-q^{10} \\
& \begin{array}{lll}
3 & 1 & 1+q+q^{2}+q^{3}-q^{4}
\end{array} \\
& 21+q+2 q^{2}+2 q^{3}+q^{4}+q^{5}+q^{6} \\
& 31+q+2 q^{2}+3 q^{3}+2 q^{4}+2 q^{5}+3 q^{6}+2 q^{7}+q^{8}+q^{9} \\
& 41+q+2 q^{2}+3 q^{3}+3 q^{4}+3 q^{5}+4 q^{6}+3 q^{7}+3 q^{8}+2 q^{9}+q^{10}+q^{12} \\
& 51+q+2 q^{2}+3 q^{3}+3 q^{4}+4 q^{5}+5 q^{6}+4 q^{7}+4 q^{8}+3 q^{9}+2 q^{10}-q^{13} \\
& -q^{14}-q^{15}-q^{16}-q^{17} \\
& 4 \quad 2 \quad 1+q+2 q^{2}+2 q^{3}+2 q^{4}+q^{5}+q^{6}-q^{9} \\
& 31+q+2 q^{2}+3 q^{3}+3 q^{4}+2 q^{5}+4 q^{6}+3 q^{7}+3 q^{8}+2 q^{9}+q^{10}+q^{11} \\
& +q^{12} \\
& 4 \quad 1+q+2 q^{2}+3 q^{3}+4 q^{4}+3 q^{5}+5 q^{6}+5 q^{7}+6 q^{8}+5 q^{9}+5 q^{10}+3 q^{11} \\
& +4 q^{12}+3 q^{13}+2 q^{14}+q^{15}+q^{16} \\
& 51+q+2 q^{2}+3 q^{3}+4 q^{4}+4 q^{5}+6 q^{6}+6 q^{7}+8 q^{8}+7 q^{9}+8 q^{10}+6 q^{11} \\
& +6 q^{12}+5 q^{13}+5 q^{14}+3 q^{15}+3 q^{16}+q^{17}+q^{18}+q^{20} \\
& 61+q+2 q^{2}+3 q^{3}+4 q^{4}+4 q^{5}+7 q^{6}+7 q^{7}+9 q^{8}+9 q^{9}+10 q^{10}+8 q^{11} \\
& +9 q^{12}+6 q^{13}+6 q^{14}+4 q^{15}+3 q^{16}-q^{19}-2 q^{20}-2 q^{21}-2 q^{22}-2 q^{23} \\
& -q^{24}-q^{25}-q^{26}
\end{aligned}
$$

I wish to acknowledge Dennis Stanton's contribution to this paper in the form of many fruitful discussions.

\section{Partitions with prescribed hook differences}

Given a partition $\lambda$ whose $i$ th largest part is $\lambda_{i}$, we define $\lambda_{i}^{\prime}$ to be the $i$ th largest part in the conjugate partition $\left(\lambda_{i}^{\prime}\right.$ is the number of parts that are greater than or equal to $i$ ). We say that $\lambda$ fits inside an $m \times n$ rectangle if $m \geq \lambda_{1}^{\prime}$ and $n \geq \lambda_{1}$. If $(i, j) \in \lambda$ (equivalently, if $\lambda_{i} \geq j$ ), then we define the hook difference at position $(i, j)$ to be $\lambda_{i}-\lambda_{j}^{\prime}$. The diagonal $\delta$ is the set of all positions $(i, j) \in \lambda$ for which $i-j=\delta$. The following proposition is a special case of theorem $1 \mathrm{in}$ $[2]$.

Proposition 1 If $-K+\beta \leq n-m \leq K-\alpha$ where $\alpha$, $\beta$, and $K$ are positive integers, $\alpha+\beta<2 K$, then $G(\alpha, \beta, K ; q)$ as defined in equation (7) is the generating function for partitions inside an $m \times n$ rectangle for which the hook differences on diagonal $\alpha-1$ are less than or equal to $K-\alpha-1$ and the hook differences on diagonal $1-\beta$ are greater than or equal to $\beta+1-K$. 
The proof of this proposition given in [2] relies on recurrences and does not lend itself to non-integer values of $\alpha$ or $\beta$. However, as we shall demonstrate, there is a combinatorial proof of this proposition that uses the approach of [3]. It is this proof that appears to be amenable to generalization.

Proof: We shall use the Frobenius representation of a partition,

$$
\lambda=\left(\begin{array}{cccc}
a_{1}, & a_{2}, & \ldots, & a_{t} \\
b_{1}, & b_{2}, & \ldots, & b_{t}
\end{array}\right)
$$

where $a_{i}=\lambda_{i}-i, b_{i}=\lambda_{i}^{\prime}-i$, and $t$ is the largest integer for which $\lambda_{t} \geq t$. We note that $a_{1}>a_{2}>\cdots>a_{t} \geq 0, b_{1}>b_{2}>\cdots>b_{t} \geq 0$, and the number being partitioned is $t+\sum\left(a_{i}+b_{i}\right)$. We want to show that $G(\alpha, \beta, K ; q)$ is the generating function for partitions whose Frobenius representation satisfies

$$
\begin{aligned}
a_{1}<n, & b_{1}<m \\
a_{i}-b_{i-\alpha+1} \leq K-2 \alpha, & b_{i}-a_{i-\beta+1} \leq K-2 \beta, \quad \text { for all } i .
\end{aligned}
$$

We shall say that a partition has an $(\alpha, \beta, K)$ positive oscillation of length $j, j \geq 1$, if we can find a sequence $i_{1}<i_{2}<\cdots<i_{j}$ for which

$$
\begin{aligned}
& a_{i_{1}}-b_{i_{1}-\alpha+1}>K-2 \alpha, \\
& b_{i_{2}}-a_{i_{2}-\beta+1}>K-2 \beta, \\
& \vdots \\
& \left\{\begin{array}{c}
a_{i_{j}}-b_{i_{j}-\alpha+1}>K-2 \alpha, \quad j \text { odd, } \\
b_{i_{j}}-a_{i_{j}-\beta+1}>K-2 \beta, \quad j \text { even. }
\end{array}\right.
\end{aligned}
$$

A partition has an $(\alpha, \beta, K)$ negative oscillation of length $j, j \geq 1$, if we can find a sequence $i_{1}<i_{2}<\cdots<i_{j}$ for which

$$
\begin{aligned}
& b_{i_{1}}-a_{i_{1}-\beta+1}>K-2 \beta, \\
& a_{i_{2}}-b_{i_{2}-\alpha+1}>K-2 \alpha, \\
& \vdots \\
& \left\{\begin{array}{cc}
a_{i_{j}}-b_{i_{j}-\alpha+1}>K-2 \alpha, & j \text { even, } \\
b_{i_{j}}-a_{i_{j}-\beta+1}>K-2 \beta, & j \text { odd. }
\end{array}\right.
\end{aligned}
$$

Lemma 1 If $\alpha, \beta$, and $K$ are positive integers with $\alpha+\beta<2 K$ and if $-K+\beta \leq$ $n-m \leq K-\alpha$, then the generating function for partitions inside an $m \times n$ rectangle with an $(\alpha, \beta, K)$ positive oscillation of length $j$ is

$$
f_{\alpha, \beta, K}^{+}(j ; q)=q^{j[K(\alpha+\beta) j+K(\alpha-\beta)] / 2}\left[\begin{array}{c}
m+n \\
m+K j
\end{array}\right] .
$$


By conjugating the partition (interchanging the $a$ s and $b$ s in the Frobenius representation), this lemma implies that the generating function for partitions inside an $m \times n$ rectangle with an $(\alpha, \beta, K)$ negative oscillation of length $j$ is

$$
f_{\alpha, \beta, K}^{-}(j ; q)=q^{j[K(\alpha+\beta) j-K(\alpha-\beta)] / 2}\left[\begin{array}{c}
m+n \\
m-K j
\end{array}\right] .
$$

Lemma 1 thus implies that

$$
G(\alpha, \beta, K ; q)=\left[\begin{array}{c}
m+n \\
m
\end{array}\right]+\sum_{j=1}^{\infty}(-1)^{j}\left[f_{\alpha, \beta, K}^{+}(j ; q)+f_{\alpha, \beta, K}^{-}(j ; q)\right] .
$$

Proposition 1 is an immediate consequence of equation (15): $\left[\begin{array}{c}m+n \\ m\end{array}\right]$ is the generating function for all partitions that sit inside an $m \times n$ box, and if such a partition has a positive or negative oscillation and if $j$ is the length of the longest such oscillation, then the alternating sum will count it with a total weight of

$$
-2\left\lfloor\frac{j}{2}\right\rfloor+2\left\lfloor\frac{j-1}{2}\right\rfloor+(-1)^{j}=-1
$$

Proof of lemma 1: Let $\lambda$ be a partition into at most $m+K j$ parts, each part less than or equal to $n-K j$. If $i$ is greater than the number of parts in $\lambda$, then we define $\lambda_{i}=0$. We let $t$ be the largest integer $(\geq 0)$ such that

$$
\lambda_{2\lceil j / 2\rceil \alpha+2\lfloor j / 2\rfloor \beta+t} \geq t,
$$

and then define sequences $a_{1}, \ldots, a_{\mu}$ and $b_{1}, \ldots, b_{\mu}, \mu=\lceil j / 2\rceil \alpha+\lfloor j / 2\rfloor \beta+t$, as follows. If $j$ is even, then

$a_{i}=\left\{\begin{array}{lr}\lambda_{i}+j K-i, & 1 \leq i \leq \alpha \\ \lambda_{i+\alpha+\beta}+(j-2) K+\alpha+\beta-i, & \alpha+1 \leq i \leq 2 \alpha+\beta, \\ \lambda_{i+2(\alpha+\beta)}+(j-4) K+2(\alpha+\beta)-i, & 2 \alpha+\beta+1 \leq i \leq 3 \alpha+2 \beta \\ \vdots & j(\alpha+\beta) / 2-\beta+1 \leq i \leq j(\alpha+\beta) / 2+t\end{array}\right.$

$b_{i}=\left\{\begin{array}{lr}\lambda_{\alpha+i}+(j-1) K+\alpha-i, & 1 \leq i \leq \alpha+\beta, \\ \lambda_{2 \alpha+\beta+i}+(j-3) K+2 \alpha+\beta-i, & \alpha+\beta+1 \leq i \leq 2(\alpha+\beta), \\ \lambda_{3 \alpha+2 \beta+i}+(j-5) K+3 \alpha+2 \beta-i, & 2(\alpha+\beta)+1 \leq i \leq 3(\alpha+\beta), \\ \vdots & \\ \lambda_{j(\alpha+\beta) / 2-\beta+i}+K+j(\alpha+\beta) / 2-\beta-i, & \left(\frac{j}{2}-1\right)(\alpha+\beta)+1 \leq i \leq j(\alpha+\beta) / 2, \\ \lambda_{i-j(\alpha+\beta) / 2}^{\prime}-j(\alpha+\beta) / 2-i, & j(\alpha+\beta) / 2+1 \leq i \leq j(\alpha+\beta) / 2+t .\end{array}\right.$ 
We note that

$$
\begin{aligned}
\frac{j}{2}(\alpha+\beta)+t+\sum_{i}\left(a_{i}+b_{i}\right) & =\sum_{i=1}^{j(\alpha+\beta)+t} \lambda_{i}+\sum_{i=1}^{t} \lambda_{i}^{\prime}-t[j(\alpha+\beta)+t] \\
& +\frac{j}{2}[K(\alpha+\beta) j+K(\alpha-\beta)] \\
& =\sum_{i \geq 1} \lambda_{i}+\frac{j}{2}[K(\alpha+\beta) j+K(\alpha-\beta)] .
\end{aligned}
$$

If $j$ is odd then,

$$
\begin{aligned}
& a_{i}=\left\{\begin{array}{lr}
\lambda_{i}+j K-i, & 1 \leq i \leq \alpha, \\
\lambda_{i+\alpha+\beta}+(j-2) K+\alpha+\beta-i, & \alpha+1 \leq i \leq 2 \alpha+\beta, \\
\vdots & \\
\lambda_{i+(j-1)(\alpha+\beta) / 2}+K+(j-1)(\alpha+\beta) / 2-i, & \\
\quad(j-1)(\alpha+\beta) / 2-\beta+1 \leq i \leq \alpha+(j-1)(\alpha+\beta) / 2, & \\
\lambda_{i-\alpha-(j-1)(\alpha+\beta) / 2}^{\prime}-\alpha-(j-1)(\alpha+\beta) / 2-i, & \\
& \alpha+(j-1)(\alpha+\beta) / 2+1 \leq i \leq \alpha+(j-1)(\alpha+\beta) / 2+t,
\end{array}\right. \\
& b_{i}=\left\{\begin{array}{lr}
\lambda_{\alpha+i}+(j-1) K+\alpha-i, & 1 \leq i \leq \alpha+\beta, \\
\lambda_{2 \alpha+\beta+i}+(j-3) K+2 \alpha+\beta-i, & \alpha+\beta+1 \leq i \leq 2(\alpha+\beta), \\
\vdots & \\
\lambda_{\alpha+(j-1)(\alpha+\beta) / 2+i}+\alpha+(j-1)(\alpha+\beta) / 2-i & (j-1)(\alpha+\beta) / 2+1 \leq i \leq(j-1)(\alpha+\beta) / 2+\alpha+t
\end{array}\right.
\end{aligned}
$$

Here we have that

$$
\begin{aligned}
\alpha+\frac{j-1}{2}(\alpha+\beta)+t+\sum_{i}\left(a_{i}+b_{i}\right)= & \sum_{i=1}^{(j+1) \alpha+(j-1) \beta+t} \lambda_{i}+\sum_{i=1}^{t} \lambda_{i}^{\prime} \\
& -t[(j+1) \alpha+(j-1) \beta+t] \\
& +\frac{j}{2}[K(\alpha+\beta) j+K(\alpha-\beta)] \\
= & \sum_{i \geq 1} \lambda_{i}+\frac{j}{2}[K(\alpha+\beta) j+K(\alpha-\beta)] .
\end{aligned}
$$

The $\alpha_{i}$ and $\beta_{i}$ are non-negative integers because of the choice of $t$. Since $\lambda_{1} \leq n-j K$ and $n-m \leq K-\alpha$, we have that

$$
a_{1}=\lambda_{1}+j K-1<n
$$


THE ELECTRONiC JOURNAL OF COMBINATORICS 3 (2) (1996), \#R4

$$
b_{1}=\lambda_{\alpha+1}+(j-1) K+\alpha-1<m .
$$

Furthermore, if we take $i_{1}=\alpha, i_{2}=\alpha+\beta, i_{3}=2 \alpha+\beta, i_{4}=2 \alpha+2 \beta$, $\ldots, i_{j}=\lceil j / 2\rceil \alpha+\lfloor j / 2\rfloor \beta$, then these sequences satisfy the inequalities in (11) that characterize a partition with an $(\alpha, \beta, K)$ positive oscillation of length $j$. The only reason why these sequences might not represent a partition with an $(\alpha, \beta, K)$ positive oscillation of length $j$ is that we might have

$$
b_{j(\alpha+\beta) / 2} \leq b_{j(\alpha+\beta) / 2+1}
$$

when $j$ is even or

$$
a_{\alpha+(j-1)(\alpha+\beta) / 2} \leq a_{\alpha+(j-1)(\alpha+\beta) / 2+1}
$$

when $j$ is odd.

\subsection{Combinatorial proof of equivalence: first direction}

We now perform a shifting operation that is done, successively, for each integer value of $r$ from $j$ down through 1 . Initially, we take $i_{j+1}$ to be $\infty, i_{r}=\lceil r / 2\rceil \alpha+$ $\lfloor r / 2\rfloor \beta$ for $j \geq r \geq 1$. Our objective is to define a bijection between the pairs of sequences given above and the pairs of sequences that give the Frobenius representation for partitions inside an $m \times n$ rectangle with an $(\alpha, \beta, K)$ positive oscillation of length $j$.

If $r$ is even:

$$
\begin{aligned}
\tau & =b_{i_{r}}-a_{i_{r}-\beta+1}, \\
\kappa & =\max \left\{\nu \mid i_{r}<\nu \leq i_{r+1}-\alpha, b_{\nu}-a_{\nu-\beta+1}>\tau\right\}, \\
\gamma(\nu) & =\max \left\{b_{i}-a_{i-\beta+1}-\tau \mid \nu \leq i \leq \kappa\right\} .
\end{aligned}
$$

If the set that defines $\kappa$ is empty, then we do no shifting for this value of $r$. Otherwise, for $i_{r}<\nu \leq \kappa$, we set

$$
\begin{aligned}
b_{\nu} & \longleftarrow b_{\nu}-\gamma(\nu), \\
a_{\nu-\beta} & \longleftarrow a_{\nu-\beta}+\gamma(\nu),
\end{aligned}
$$

and then reset the value of $i_{r}$ to $\kappa$.

If $r$ is odd:

$$
\begin{aligned}
\tau & =a_{i_{r}}-b_{i_{r}-\alpha+1}, \\
\kappa & =\max \left\{\nu \mid i_{r}<\nu \leq i_{r+1}-\beta, a_{\nu}-b_{\nu-\alpha+1}>\tau\right\}, \\
\gamma(\nu) & =\max \left\{a_{i}-b_{i-\alpha+1}-\tau \mid \nu \leq i \leq \kappa\right\} .
\end{aligned}
$$


If the set that defines $\kappa$ is empty, then we do no shifting for this value of $r$. Otherwise, for $i_{r}<\nu \leq \kappa$, we set

$$
\begin{aligned}
a_{\nu} & \longleftarrow a_{\nu}-\gamma(\nu), \\
b_{\nu-\alpha} & \longleftarrow b_{\nu-\alpha}+\gamma(\nu),
\end{aligned}
$$

and then reset the value of $i_{r}$ to $\kappa$.

We claim that this shifting procedure yields a pair of sequences that give the Frobenius representation for a partition inside an $m \times n$ rectangle with an $(\alpha, \beta, K)$ positive oscillation of length $j$.

If $j$ is even, then after doing the shift for $r=j$, the new value of $b_{j(\alpha+\beta) / 2}$ is strictly larger than the new value of $b_{j(\alpha+\beta) / 2+1}$. To see this, we observe that the value of $b_{j(\alpha+\beta) / 2}$ does not change, and if the old value of $b_{j(\alpha+\beta) / 2+1}$ is greater than or equal to the old value of $b_{j(\alpha+\beta) / 2}$, then

$\gamma(j(\alpha+\beta) / 2+1) \geq b_{j(\alpha+\beta) / 2+1}-a_{j(\alpha+\beta) / 2-\beta+2}-\left(b_{j(\alpha+\beta) / 2}-a_{j(\alpha+\beta) / 2-\beta+1}\right)$,

so that the new value of $b_{j(\alpha+\beta) / 2+1}$ equals

$$
\begin{aligned}
b_{j(\alpha+\beta) / 2+1}-\gamma(j(\alpha+\beta) / 2+1) & \leq b_{j(\alpha+\beta) / 2}+a_{j(\alpha+\beta) / 2-\beta+2}-a_{j(\alpha+\beta) / 2-\beta+1} \\
& <b_{j(\alpha+\beta) / 2} .
\end{aligned}
$$

The function $\gamma$ is weakly decreasing, and so the new values of $a_{\nu-\beta}$ are still strictly decreasing. We do need to verify that

$$
b_{\nu}-\gamma(\nu)>b_{\nu+1}-\gamma(\nu+1) .
$$

This will be true if $\gamma(\nu)=\gamma(\nu+1)$. If these values of $\gamma$ are not equal, then by definition:

$$
\gamma(\nu)=b_{\nu}-a_{\nu-\beta+1}-\tau, \quad \gamma(\nu+1) \geq b_{\nu+1}-a_{\nu-\beta+2}-\tau .
$$

It follows that

$$
b_{\nu}-\gamma(\nu)=a_{\nu-\beta+1}+\tau>a_{\nu-\beta+2}+\tau \geq b_{\nu+1}-\gamma(\nu+1) .
$$

We note that after the shift for $r=j$, the value of $a_{j(\alpha+\beta) / 2-\beta}$ might be less than or equal to the new value of $a_{j(\alpha+\beta) / 2-\beta+1}$ which will be bounded by

$$
a_{j(\alpha+\beta) / 2-\beta+1}<\lambda_{1}^{\prime}-j \alpha-(j-2) \beta-K .
$$

After the next shift, for $r=j-1$, the new value of $a_{j(\alpha+\beta) / 2-\beta}$ will be strictly greater than the new value of $a_{j(\alpha+\beta) / 2-\beta+1}$.

The same argument holds mutatis mutandis if $j$ is odd and for each successive value of $r$. If $r$ is even, then the new value of $a_{\lceil r / 2\rceil \alpha+\lfloor r / 2\rfloor \beta-\beta+1}$ is bounded by

$$
a_{\lceil r / 2\rceil \alpha+\lfloor r / 2\rfloor \beta-\beta+1}<\lambda_{1}^{\prime}-r \alpha-(r-2) \beta-(j-r+1) K .
$$


If $r$ is odd, then the new value of $b_{\lceil r / 2\rceil \alpha+\lfloor r / 2\rfloor \beta-\alpha+1}$ is bounded by

$$
b_{\lceil r / 2\rceil \alpha+\lfloor r / 2\rfloor \beta-\alpha+1}<\lambda_{1}^{\prime}-(r-1) \alpha-(r-1) \beta-(j-r+1) K .
$$

We observe that the value of $a_{1}$ is left unchanged and so is strictly less than $n$, and that the final value of $b_{1}$ (after the shift that corresponds to $r=1$ ) is strictly less than $\lambda_{1}^{\prime}-j K \leq m$.

\subsection{Combinatorial proof of equivalence: other direction}

To see that we do, in fact, have a bijection we note that we can uniquely reconstruct the values of $\tau, \kappa$, and $\gamma(\nu)$ for each shift as $r$ runs from 1 back up to $j$. We first choose the sequence $i_{1}<i_{2}<\cdots<i_{j}$ maximally. That is to say, we find the largest integer $i_{j}$ for which $b_{i_{j}}-a_{i_{j}-\beta+1}>K-2 \beta$ (if $j$ is even) or $a_{i_{j}}-b_{i_{j}-\alpha+1}>K-2 \alpha$ (if $j$ is odd), and then after each $i_{r}$ is chosen, we choose the largest possible value for $i_{r-1}$. To reverse the shifting process, we perform the following operation for each $r$ from 1 through $j$.

\section{If $r$ is even:}

$$
\begin{aligned}
\tau^{*} & =\max \left\{b_{\nu}-a_{\nu-\beta+1} \mid r(\alpha+\beta) / 2 \leq \nu \leq i_{r}\right\}, \\
\kappa^{*} & =\min \left\{\nu \mid r(\alpha+\beta) / 2 \leq \nu \leq i_{r}, b_{\nu}-a_{\nu-\beta+1}=\tau^{*}\right\}, \\
\gamma^{*}(\nu) & =\min \left\{\tau^{*}-\left(b_{i}-a_{i-\beta+1}\right) \mid r(\alpha+\beta) / 2 \leq i<\nu\right\} .
\end{aligned}
$$

For $r(\alpha+\beta) / 2<\nu \leq \kappa^{*}$, we set

$$
\begin{aligned}
b_{\nu} & \longleftarrow b_{\nu}+\gamma^{*}(\nu), \\
a_{\nu-\beta} & \longleftarrow a_{\nu-\beta}-\gamma^{*}(\nu) .
\end{aligned}
$$

If $r$ is odd:

$$
\begin{aligned}
\tau^{*} & =\max \left\{a_{\nu}-b_{\nu-\alpha+1} \mid\lceil r / 2\rceil \alpha+\lfloor r / 2\rfloor \beta \leq \nu \leq i_{r}\right\}, \\
\kappa^{*} & =\min \left\{\nu \mid\lceil r / 2\rceil \alpha+\lfloor r / 2\rfloor \beta \leq \nu \leq i_{r}, a_{\nu}-b_{\nu-\alpha+1}=\tau^{*}\right\}, \\
\gamma^{*}(\nu) & =\min \left\{\tau^{*}-\left(a_{i}-b_{i-\alpha+1}\right) \mid\lceil r / 2\rceil \alpha+\lfloor r / 2\rfloor \beta \leq i<\nu\right\} .
\end{aligned}
$$

For $\lceil r / 2\rceil \alpha+\lfloor r / 2\rfloor \beta<\nu \leq \kappa^{*}$, we set

$$
\begin{aligned}
a_{\nu} & \longleftarrow a_{\nu}+\gamma^{*}(\nu), \\
b_{\nu-\alpha} & \longleftarrow b_{\nu-\alpha}-\gamma^{*}(\nu) .
\end{aligned}
$$

It is left to the reader to verify that this does uniquely reverse the shifting done in section 2.1. To prove that we have a bijection between pairs of sequences generated by $f_{\alpha, \beta, K}^{+}(j ; q)$ and partitions inside an $m \times n$ rectangle with an $(\alpha, \beta, K)$ positive oscillation of lenth $j$, we need to verify that if we start with 
an arbitrary partition, we get a pair of sequences generated by $f_{\alpha, \beta, K}^{+}(j ; q)$. The only condition on these sequences that is not straightforward to verify is that they are strictly decreasing with the possible exception that if $j$ is even then we might have $b_{j(\alpha+\beta) / 2+1} \geq b_{j(\alpha+\beta) / 2}$ and if $j$ is odd then we might have $a_{\lceil j / 2\rceil \alpha+\lfloor j / 2\rfloor \beta+1} \geq a_{\lceil j / 2\rceil \alpha+\lfloor j / 2\rfloor \beta}$.

We observe that in applying the shift given above for $r=1$, the new value for $a_{\alpha}$ might be less than or equal to the new value for $a_{\alpha+1}$. If $j=1$, there is no problem. If $j$ is larger than 1 , then on the $r=2$ shift we replace $a_{\alpha+1}$ with

$$
a_{\alpha+1} \longleftarrow a_{\alpha+1}-\left(\tau^{*}-b_{\alpha+\beta}+a_{\alpha+1}\right)=b_{\alpha+\beta}-\tau^{*} .
$$

We note that if the new value of $a_{\alpha+1}$ is greater than or equal to the new value of $a_{\alpha}$, then the value of $b_{1}$ after the $r=1$ shift is strictly less than its original value. This implies that after the $r=1$ shift we have $a_{\alpha}-b_{1}>K-2 \alpha$. We combine this observation with the following inequalities:

$$
\begin{aligned}
b_{1}-b_{\alpha+\beta} & \geq \alpha+\beta-1, \\
\tau^{*} & \geq K-2 \beta \\
2 K & >\alpha+\beta
\end{aligned}
$$

to see that

$$
b_{\alpha+\beta}-\tau^{*}<a_{\alpha} .
$$

The new value of $a_{\alpha+1}$ after the $r=2$ shift is strictly less than $a_{\alpha}$. This argument continues to hold for each $r<j$ so that after all of the shifting we have at most one pair of consecutive elements in the sequences for which we do not have strict decrease.

Q.E.D.

\section{Prescribed Hook Differences with non-integer parameters}

We want to define a prescribed hook difference condition when $\alpha$ and $\beta$ are not integers. While there does not seem to be hope for doing this in general, the particular case

$$
\alpha=\nu+\frac{K+1}{2}-\frac{a}{K}, \quad \beta=-\nu+\frac{K-1}{2}+\frac{a}{K}
$$

does hold promise. In particular, let

$$
\alpha=\bar{\alpha}-a / K, \quad \beta=\bar{\beta}+a / K
$$


where $\bar{\alpha}$ and $\bar{\beta}$ are positive integers and $a$ is a positive integer less than or equal to $\min \{\bar{\alpha}, \bar{\beta}\}$. Let $\left\{\bar{a}_{i}\right\}$ and $\left\{\bar{b}_{i}\right\}$ be the pair of sequences generated by

$$
f_{\bar{\alpha}, \bar{\beta}, K}^{+}(j ; q)=q^{j[K(\bar{\alpha}+\bar{\beta}) j+K(\bar{\alpha}-\bar{\beta})] / 2}\left[\begin{array}{c}
m+n \\
m+K j
\end{array}\right]
$$

as given in equations (17-18) and (20-21). We now define

$$
\begin{aligned}
& a_{i}=\bar{a}_{i}- \begin{cases}1, & \text { if } \bar{\alpha}-a+1 \leq(i \bmod \bar{\alpha}+\bar{\beta}) \leq \bar{\alpha} \\
& \text { and } i \leq\lceil j / 2\rceil(\bar{\alpha}+\bar{\beta})-\bar{\beta}, \\
0, & \text { otherwise }\end{cases} \\
& b_{i}=\bar{b}_{i}-\left\{\begin{array}{cc}
1, & \text { if } \bar{\alpha}+\bar{\beta}-a+1 \leq(i \bmod \bar{\alpha}+\bar{\beta}) \leq \bar{\alpha}+\bar{\beta}, \\
& \text { and } i \leq\lfloor j / 2\rfloor(\bar{\alpha}+\bar{\beta}), \\
0, & \text { otherwise, }
\end{array}\right.
\end{aligned}
$$

where $(i \bmod \bar{\alpha}+\bar{\beta})$ is the least positive residue of $i$ modulo $(\bar{\alpha}+\bar{\beta}=\alpha+\beta)$. We have subtracted a total of $a j$ from the pair of sequences. We are left with a pair of sequences generated by

$$
f_{\alpha, \beta, K}^{+}(j ; q)=q^{j[K(\alpha+\beta) j+K(\alpha-\beta)] / 2}\left[\begin{array}{c}
m+n \\
m+K j
\end{array}\right] .
$$

To get a pair of sequences generated by $f_{\alpha, \beta, K}^{-}(j ; q)$, we find the sequences generated by $f_{\beta, \alpha, K}^{+}(j ; q)$ and then interchange the $a$ s and $b$ s. This means that we start with the pair of sequences generated by $f_{\bar{\beta}, \bar{\alpha}, K}^{+}(j ; q)$ and then add $a j$ to them by defining

$$
\begin{aligned}
& a_{i}=\bar{a}_{i}+\left\{\begin{aligned}
1, & \text { if } \bar{\beta}+1 \leq(i \bmod \bar{\beta}+\bar{\alpha}) \leq \bar{\beta}+a \\
& \text { and } i \leq\lfloor j / 2\rfloor(\bar{\beta}+\bar{\alpha}), \\
0, & \text { otherwise, }
\end{aligned}\right. \\
& b_{i}=\bar{b}_{i}+\left\{\begin{array}{rr}
1, & \text { if } 1 \leq(i \bmod \bar{\beta}+\bar{\alpha}) \leq a, \\
& \text { and } i \leq\lceil j / 2\rceil \bar{\beta}+\lfloor j / 2\rfloor \bar{\alpha}, \\
0, & \text { otherwise. }
\end{array}\right.
\end{aligned}
$$

It is not clear to what partitions these pairs of sequences correspond. The sequences generated by $f_{\alpha, \beta, K}^{+}(j ; q)$ satisfy a weakened form of the oscillating condition. If we set $i_{r}=\lceil r / 2\rceil \bar{\alpha}+\lfloor r / 2\rfloor \bar{\beta}$, then

$$
\begin{array}{ll}
a_{i_{r}}-b_{i_{r}-\bar{\alpha}+1} \geq K-2 \bar{\alpha}, & j \text { odd }, \\
b_{i_{r}}-a_{i_{r}-\bar{\beta}+1} \geq K-2 \bar{\beta}, & j \text { even. }
\end{array}
$$

We also introduce additional inequalities:

$$
\begin{array}{ll}
a_{i_{r}-a}>a_{i_{r}-a+1}, & j \text { odd, } \\
b_{i_{r}-a}>b_{i_{r}-a+1}, & j \text { even. }
\end{array}
$$




\section{1 $\mathcal{A}_{m, n}(q)$ : a related partition generating function}

If we restrict our attention to the polynomial $A_{n}(q)=G(5 / 3,4 / 3,3 ; q)$ given in conjecture 1 - see equations (2) and (7) - then we have

$$
K=3, \quad a=1, \quad, \bar{\alpha}=2, \quad \bar{\beta}=1 .
$$

A family of partitions whose generating function appears to be very closely related to $A_{n}(q)$ consists of those that fit inside an $m \times n$ rectangle and satisfy the following conditions for all $i$ such that $\lambda_{i} \geq i$ :

$$
\begin{array}{lll}
\text { either } & \lambda_{i}-\lambda_{i}^{\prime} \geq 0 & \text { or } \quad \lambda_{i}=\lambda_{i+1}, \\
\text { either } & \lambda_{i+1}-\lambda_{i}^{\prime} \leq-1 & \text { or } \quad \lambda_{i}=\lambda_{i+1} .
\end{array}
$$

These two conditions can be combined into

$$
\text { either } \quad \lambda_{i}=\lambda_{i+1} \quad \text { or } \quad \lambda_{i} \geq \lambda_{i}^{\prime}>\lambda_{i+1} .
$$

If we designate the generating function for these partitions by $\mathcal{A}_{m, n}(q)$, then we have a simple recursion from which they can be computed:

$$
\begin{aligned}
\mathcal{A}_{m, n}= & 0, \quad \text { if } m<0 \quad \text { or } \quad n<0, \\
\mathcal{A}_{m, n}= & 1, \quad \text { if } m n=0, \quad m \geq 0, n \geq 0 \\
\mathcal{A}_{1, n}= & {\left[\begin{array}{c}
n+1 \\
1
\end{array}\right], \quad \text { if } n \geq 0, } \\
\mathcal{A}_{m, n}= & \mathcal{A}_{m-1, n}+\mathcal{A}_{m, n-1}-\mathcal{A}_{m-1, n-1} \\
& +q^{m+n-1}\left[\mathcal{A}_{m-1, n-1}-\mathcal{A}_{m-1, n-2}+\chi(m \leq n) \mathcal{A}_{m-1, m-2}\right], \\
& \quad \text { if } m \geq 2, \quad n \geq 1 .
\end{aligned}
$$

We compare $\mathcal{A}_{n, n}$ with $A_{n}$ :

$$
\begin{aligned}
A_{n}(q)-\mathcal{A}_{n, n}(q)= & 0, \quad n \leq 4, \\
A_{5}(q)-\mathcal{A}_{5,5}(q)= & q^{11}-q^{12}-q^{13}+q^{14} \\
A_{6}(q)-\mathcal{A}_{6,6}(q)= & q^{11}-q^{13}-q^{15}+q^{17}+q^{19}-q^{21}-q^{23}+q^{25}, \\
A_{7}(q)-\mathcal{A}_{7,7}(q)= & q^{11}-q^{15}-q^{16}+q^{19}+q^{20}+2 q^{22}-q^{23}-2 q^{24} \\
& -2 q^{25}-q^{26}+2 q^{27}+q^{29}+q^{30}-q^{33}-q^{34}+q^{38} .
\end{aligned}
$$

It is easily verified by induction that

$$
\mathcal{A}_{m, n}(1)= \begin{cases}2 \times 3^{n-1}, & m=n, \\ 3^{\min (m, n)}, & |m-n|=1 .\end{cases}
$$

so that in fact

$$
A_{n}(1)=\mathcal{A}_{n, n}(1)
$$


As $n$ increases, the coefficients of $A_{n}(q)-\mathcal{A}_{n, n}(q)$ do increase, but remain substantially less than the coefficients of either $A_{n}(q)$ or $\mathcal{A}_{n, n}(q)$. Plots of these coefficients for $n=8,10,12,15$, and 18 are included in the file borwein.ps. While we do get coefficients on the order of 4000 when $n=18$, this is less than $1 / 500$ of the corresponding coefficient in either $A_{n}(q)$ or $\mathcal{A}_{n, n}(q)$.

One interesting pattern does emerge. For $5 \leq n \leq 17$,

$$
P_{n}(q)=\frac{A_{n}(q)-\mathcal{A}_{n, n}(q)}{q^{11}(1-q)\left(1-q^{2}\right)}
$$

is a symmetric, unimodal, monic polynomial of degree $n^{2}-25$ with strictly positive coefficients. The fact that it is symmetric and monic of degree $n^{2}-25$ follows from the fact that both $\mathcal{A}_{n, n}(q)$ and $A_{n}(q)$ are symmetric polynomials. There is no apparent reason why it should be unimodal with strictly positive coefficients.

\section{References}

[1] George E. Andrews, On a conjecture of Peter Borwein, preprint.

[2] George E. Andrews et al, Partitions with prescribed hook differences, Europ. J. Combinatorics (1987) 8, 341-350.

[3] David M. Bressoud, Extension of the partition sieve, J. Number Theory (1980) 12, 87-100.

[4] David M. Bressoud, Some identities for terminating $q$ series, Math. Proc. Camb. Phil. Soc. (1981) 89, 211-223.

[5] Mourad Ismail and Dennis Stanton, private communication.

Department of Mathematics and Computer Science, Macalester College, Saint Paul, MN 55105, USA 